\title{
SPECTROSCOPIC AND PHOTOMETRIC STUDY OF STARBURST GALAXIES: OPTICAL AND NEAR INFRARED PROPERTIES OF A BLUE COMPACT DWARF GALAXY MRK 49 IN THE VIRGO CLUSTER
}

\author{
Eon-Chang Sung ${ }^{1}$, Jaemann Kyeong ${ }^{1}$, And Yong-IK Byun ${ }^{2}$ \\ ${ }^{1}$ Korea Astronomy \& Space Science Institute, Taejon 305-348, Korea \\ E-mail: ecsung,jman@kasi.re.kr \\ ${ }^{2}$ Department of Astronomy, Yonsei University, Seoul 120-749, Korea \\ E-mail: ybyun@yonsei.ac.kr \\ (Received September 30, 2008; Accepted October 17, 2008)
}

\begin{abstract}
We present optical and near-infrared imaging and long-slit spectroscopy for the blue compact dwarf galaxy (BCD) Mrk 49 in the Virgo Cluster. The surface brightness distribution analysis shows that Mrk 49 consists of an off-centered blue bright compact core of $r=10^{\prime \prime}$ and a red faint outer exponential envelope. The $H_{\alpha}$ image and color difference suggest that these two components have different stellar populations: a high surface brightness population of massive young stars and an underlying low surface brightness population of older stars. The redder near-infrared colors of the inner most region suggest that the near-infrared flux of Mrk 49 originates from evolved massive stars associated with the current star-forming activity. The total apparent magnitude is $\mathrm{B}_{T}=14.32 \mathrm{mag}$ and the mean effective surface brightness is $\mu_{\text {eff }}(B)=21.56 \mathrm{mag} \operatorname{arcsec}^{-2}$. Long-slit spectroscopy shows that Mrk 49 rotates apparently as a solid body within $r=10^{\prime \prime}$ in a plane at position angle 55 degrees with an amplitude of about $20 \mathrm{~km} \mathrm{sec}^{-1}$. The measured radial velocity of Mrk 49 was derived as 1,535 $\mathrm{km} \mathrm{sec}^{-1}$; and the total mass of stars and gases is in the range of 3 to $6 \times 10^{9} \mathrm{M}_{\odot}$. The mass-to-light ratios for the central region of Mrk 49 in $I$ and $B$ band are estimated 1.0 and 0.5 , respectively. The upper limit of the dark matter to visible matter ratio seems to be $<5$. The oxygen abundance is $12+\log (\mathrm{O} / \mathrm{H})=8.21 \pm 0.1$ which is about one quarter of the solar value while the relative helium abundance appears to be similar to that of the sun.
\end{abstract}

Key words : clusters: starburst galaxy: dwarf galaxy: blue compact dwarf galaxy: Mrk 49

\section{INTRODUCTION}

A fairly large portion of galaxy population are dwarf galaxies. They were probably more numerous in the early cosmic age. Dwarf galaxies play a crucial role in contemporary theories for the formation and evolution of galaxies. They are proposed to be the building block from which larger system have been created by merging (Driver et al. 1994, Ellis 1997, Gil de Paz, Madore \& Pevunova 2003, references therein).

At the blue extreme, blue compact dwarf galaxies (BCDs) are defined as dwarf galaxies which are characterized by low luminosities, blue continuum accompanied by intense narrow emission lines, small optical sizes, large HI mass fraction, and low oxygen abundances in their ionized interstellar gas(Meurer et al. 1992, Gil de Paz, Madore \& Pevunova 2003). On the other hand, recent studies of high- and intermediate redshift galaxies using powerful instruments like the HST and the new generation 8m-class telescopes have revealed a population of compact, luminous galaxies with narrow emission lines and high star formation rate

Corresponding Author: E.-C. Sung which have very similar observational properties to the nearby BCDs (Pisano et al. 2001, Guzman et al 1998). Thus, the evolution of BCDs over cosmic time is a central topic in dwarf galaxy research and observational cosmology.

Recent observations have confirmed that most of BCDs have underlying older stellar populations at least a few $10^{9}$ yr old (e.g., Cairós et al. 2001, Gil de Paz, Madore \& Pevunova 2003, Noeske et al. 2005). This implies that, in general, BCDs are rather old objects, and their evolution on the Hubble time scale can effectively be determined by short, but very intense star formations. On the other hand, many observations and theoretical works have recently suggested that the origin of BCDs can be closely associated with galaxy merging between low-mass dwarfs or tidal interactions (Noeske et al. 2001, Östlin et al. 2001, Sung et al. 2002, Bekki 2008).

The underlying old stellar component contains practically all stellar mass of typical BCDs, and is therefore a likely dynamically and evolutionary important constituent of BCDs. Its radial light distribution provides close constraint to the stellar mass distributions 
in a BCD, information which is crucial for modeling the global gravitational potential and dynamics of BCDs, as well as the effect of starburst events. The investigation of the evolutionary relation between different types of dwarf galaxies requires a separation of the light distributions of the young and old stellar populations. Such analysis are however hampered by the extended dominant starburst emission at visible wavelengths, and can be performed with much better precision in the near-infrared lights (Noeske et al. 2005).

Near-infrared photometric study of the class of BCDs has been carried out by Thuan(1983), Campbell \& Terlevich(1984), Hunter \& Gallagher(1985), Bothun et al.(1986), Joy \& Lester(1988), James(1991) and Quillen et al.(1995). Thuan(1983) suggested that there have been earlier star formation in addition to the present burst; they claimed that the near-infrared flux of BCDs originates from an old population of $\mathrm{K}$ and $\mathrm{M}$ giants, whose distribution is more extended than the young population. Hunter \& Gallagher(1985) also agree that the young population is not a major contribution to the near-infrared light. On the contrary, Campbell and Terlevich(1984) suggested that most of the $2 \mu \mathrm{m}$ flux in BCDs was produced by ionized gas and cool evolved stars, and the cool stellar component was red supergiants associated with the current starforming activity. Joy \& Lester(1988) presented measurements of near-infrared line and continuum emission from the BCD II Zw 40, and suggested that nebular recombination emission and photospheric radiation from young blue stars would produce most of the near infrared continuum emission in the central $6^{\prime \prime}$ of II $\mathrm{Zw}_{\mathrm{w}} 40$ and that evolved stars would contribute no more than $25 \%$ of the total $2.2 \mu \mathrm{m}$ flux in the central region of II Zw 40. Vanzi et al.(1996), however, showed that the $K$ luminosity from II $\mathrm{Zw} 40$ was due to red supergiants of older than $4 \times 10^{6}$ years. Quillen et al.(1995) show that optical and near-infrared colors in NGC 1705 are consistent with a composite population with colors similar to those of the bluest Sc nuclei. A color gradient seems to exist due to a steady decreasing fraction of young stars with increasing distance from the central part of the disk. According to Bothun et al.(1986), BCDs in Virgo Cluster display redder $J-K, B-H$ and $B-V$ colors than the more extended low-surfacebrightness dwarf irregulars (dIs) in the Virgo Cluster. The mean surface brightness of BCDs is much higher than dIs, and BCDs have lower H I content. This evidence appears to support the view that BCDs were progenitors of dwarf ellipticals(dEs). On the other hands, James(1991) presented an infrared surface photometry of dwarf galaxies in Virgo Cluster, and argued that dEs may have formed by the gas-stripping of dIs.

Although optical and near-infrared structures, HI properties, and theoretical calculations in BCDs have been discussed in terms of evolutionary links between BCDS and other type of dwarfs such as dwarf spheroidals (dSphs), dwarf ellipticals and dwarf irregulars, physical connections between these dwarfs remain unclear (van Zee et al. 1998, Sung et al. 1998, Gil de Paz et al. 2003, Noeske et al. 2005, Bekki 2008).

Mrk 49 is classified as a post-merger BCD candidate (Sung et al. 2002). Recent surveys show many dwarf galaxy mergers, but rare post-merger or merger remnants (Sung 2000, Pustilnik et al. 2001, Sung et al. 2002). A post-merger BCD is extremely important to understand the dwarf galaxy merger scenario as the origin of BCDs, and the evolutionary link between dwarf galaxies.

Mrk 49 (VCC 324, UGC 7354, Mrk 1318, Haro 8) is one of Haro's emission line galaxies. It was classified as a blue compact dwarf galaxy (BCD) in the ' $\mathrm{W}$ ' group of the Virgo Cluster by Binggeli et al.(1993). Optical, near-infrared, and radio observations for Mrk 49 have been made by Gordon \& Gottesman(1981), Thuan \& Martin(1981), Thuan(1983), Knapp et al.(1985), Gallagher \& Hunter(1986), Lake et al.(1987) and Binggeli et al.(1993). From the H I distribution of Mrk 49, Lake et al.(1987) found that the gas is centrally concentrated. However, the outer H I envelope showed a roughly circular distribution which extends 2 to 3 times its optical diameter. The $\mathrm{H}$ I velocity field does not show any systematic order, but there may be some indication of rotation with peak to peak amplitude of about $20 \mathrm{~km} \mathrm{sec}^{-1}$ along the position angle of $60 \mathrm{de}-$ gree. Their deep CCD images show two distinct structures: a bright compact elliptical component (recognized as Haro 8) and diffuse disk-like component in the outer part. From their observation, Lake et al.(1987) suggested Mrk 49 is an interacting system. From the observed radial velocity of Mrk $49\left(1,523 \mathrm{~km} \mathrm{sec}^{-1}\right)$, a distance of $18.2 \mathrm{Mpc}$ was derived by Thuan \& Martin(1981). They estimated the total mass of the galaxy as $2.8 \times 10^{8} \mathrm{M}_{\odot}$ using their neutral hydrogen observations. The $\mathrm{H}$ I mass-to-light ratio, $\mathrm{M}_{H I} / \mathrm{L}_{B}$, was observed as 0.22 (Gorden \& Gottesmann 1981, Thuan \& Martin 1981) or 0.08 (Knapp et al. 1985). This galaxy is also known as an IRAS galaxy (Parravano 1989).

The nature of the stellar population in Mrk 49, however, remains uncertain. Until now, no complete study is available for this galaxy. In our investigation, we present an extensive photometric imaging study covering optical and near-infrared wavelengths (UBVRIJK, $H_{\alpha}$ imaging) combined with a spectroscopic study of kinematic properties. Tab. 1 lists the compilation of the basic properties of Mrk 49. We adopt a distance of $18.2 \mathrm{Mpc}$ to Mrk 49 based on the linear Virgo-centric flow model of Thuan \& Martin(1981) assuming the Hubble Constant of $\mathrm{H}_{\circ}=75$ $\mathrm{km} \mathrm{sec}^{-1} \mathrm{Mpc}^{-1}$.

\section{OBSERVATIONS AND DATA REDUC- TION}

Observation logs for imaging and spectroscopic observations are given in Table 2 and 3 respectively. The first 3 columns give date, filter, and exposure time. The observations and reductions are described in detail be- 
TABLE 1.

Properties OF MrK 49

\begin{tabular}{ccc}
\hline \hline Parameter & Value & Note \\
\hline $\mathrm{RA}(\mathrm{J} 2000.0)$ & $12^{h} 19^{m} 09 .^{s} 9$ & from NED $^{\dagger}$ \\
$\mathrm{DEC}(\mathrm{J} 2000.0)$ & $03^{\circ} 51^{\prime} 28^{\prime \prime}$ & \\
$l$ & $283 .^{\circ} 209$ & \\
$b$ & $65 .^{\circ} 484$ & this paper \\
$V_{r}$ & $1535 \pm 10 \mathrm{~km} / \mathrm{sec}$ & from NED \\
$\mathrm{D}$ & $18.2 \mathrm{Mpc}$ & from NED \\
$\mathrm{A}_{B}$ & $0.02 \mathrm{mag}$ & this paper \\
$12+\log (\mathrm{O} / \mathrm{H})$ & $8.21 \pm 0.2$ & this paper \\
$\mathrm{DH}$ & 39 arcsec & from Thuan \& Martin $(1981)$ \\
$\mathrm{M}_{H}$ & $2 \times 10^{8} \mathrm{M} \odot$ & this paper \\
$M_{B}$ & $-17.03 \mathrm{mag}$ & this paper \\
$M_{I}$ & $-18.02 \mathrm{mag}$ &
\end{tabular}

$\dagger$ : The NASA/IPAC Extragalactic Database(NED) is operated by the Jet Propulsion Laboratory, California Institute of Technology, under the contract with the National Aeronautics and Space Administration.

TABLE 2.

IMAGING OBSERVATIONS

\begin{tabular}{|c|c|c|c|c|c|}
\hline Date & Filter & Exposure & Airmass & Seeing $\left({ }^{\prime \prime}\right)$ & Comments \\
\hline \multirow[t]{4}{*}{ April 151993} & $\mathrm{~B}$ & $450 \sec \times 3$ & 1.222 & 2.5 & $\mathrm{SSO} 1 \mathrm{~m}$ \\
\hline & $\mathrm{V}$ & $450 \sec \times 3$ & 1.233 & $\because$, & \\
\hline & $\mathrm{R}$ & $450 \sec \times 3$ & 1.293 & $"$ & \\
\hline & I & $300 \sec \times 3$ & 1.370 & $"$ & \\
\hline \multirow[t]{3}{*}{ March 141994} & $\mathrm{U}$ & $600 \sec \times 3$ & 1.255 & 2.0 & \\
\hline & H6585 & $500 \sec \times 2$ & 1.286 & , & \\
\hline & H6676 & $500 \sec \times 2$ & 1.254 & $"$ & \\
\hline \multirow[t]{2}{*}{151994} & B & $400 \sec \times 3$ & 1.237 & 2.0 & \\
\hline & $\mathrm{V}$ & $450 \sec \times 3$ & 1.272 & $"$ & \\
\hline \multirow[t]{2}{*}{161994} & $\mathrm{R}$ & $450 \sec \times 3$ & 1.223 & 1.5 & \\
\hline & I & $450 \sec \times 3$ & 1.286 & $"$ & \\
\hline \multirow[t]{2}{*}{ April 51993} & $\mathrm{~J}$ & $60 \sec \times 8$ & 1.04 & 1.2 & U. of Hawaii $2.2 \mathrm{~m}$ \\
\hline & $\mathrm{K}^{\prime}$ & $60 \sec \times 8$ & 1.14 & 1.2 & \\
\hline
\end{tabular}

low.

\section{(a) $U B V R I$ and $H_{\alpha}$ CCD Imaging}

The Australian National University 1-m telescope at the Siding Spring Observatory(SSO) was used on 1993 April and 1994 March. All observations were carried out with the Tek $1024 \times 1024$ pixel CCD camera placed at the $\mathrm{f} / 8$ Cassegrain focus, which yields a plate scale of $0.57 /$ pixel and a field view of $9 ! 7 \times 9 ! 7$. We used $U B V R I$ (Bessell 1990) and 2 narrow-band filters with a $50 \AA$ FWHM (centered at $6585 \AA$ ), and a $55 \AA$ FWHM (centered at $6676 \AA$ ), respectively (Ryder 1993), English 1994). The scaled 6676 filter was employed in order to subtract the stellar continuum from 6585 frame which enables us to produce a pure $\mathrm{H}_{\alpha}$ emission-line image. The contamination by [N II] emission lines is estimated to be less than $5 \%$ of the total $\mathrm{H}_{\alpha}$ emission. The typical seeing was 1".5 - 2'.2. In 1993 observation, E-region standard stars (Graham 1982), and in 1994 observation, Landolt (1992)'s standard stars were observed to place the data on the Cousin-Johnson and the Kron-Cousin system (Bessell 1990).

The images were reduced using a standard CCD reduction procedure, with the Image Reduction and Analysis Facility (IRAF) of NOAO. First, the images were corrected for zero level bias and overscan pattern. For flatfielding, we used twilight sky images. However, for $I$ band images, we had to use dark night sky flat frames because they needed fringe correction. After the flat fielding and fringe correction were done, the remaining peak-to-peak variations in the sky level were not larger than $0.2 \%$ of the sky value. The sky level 

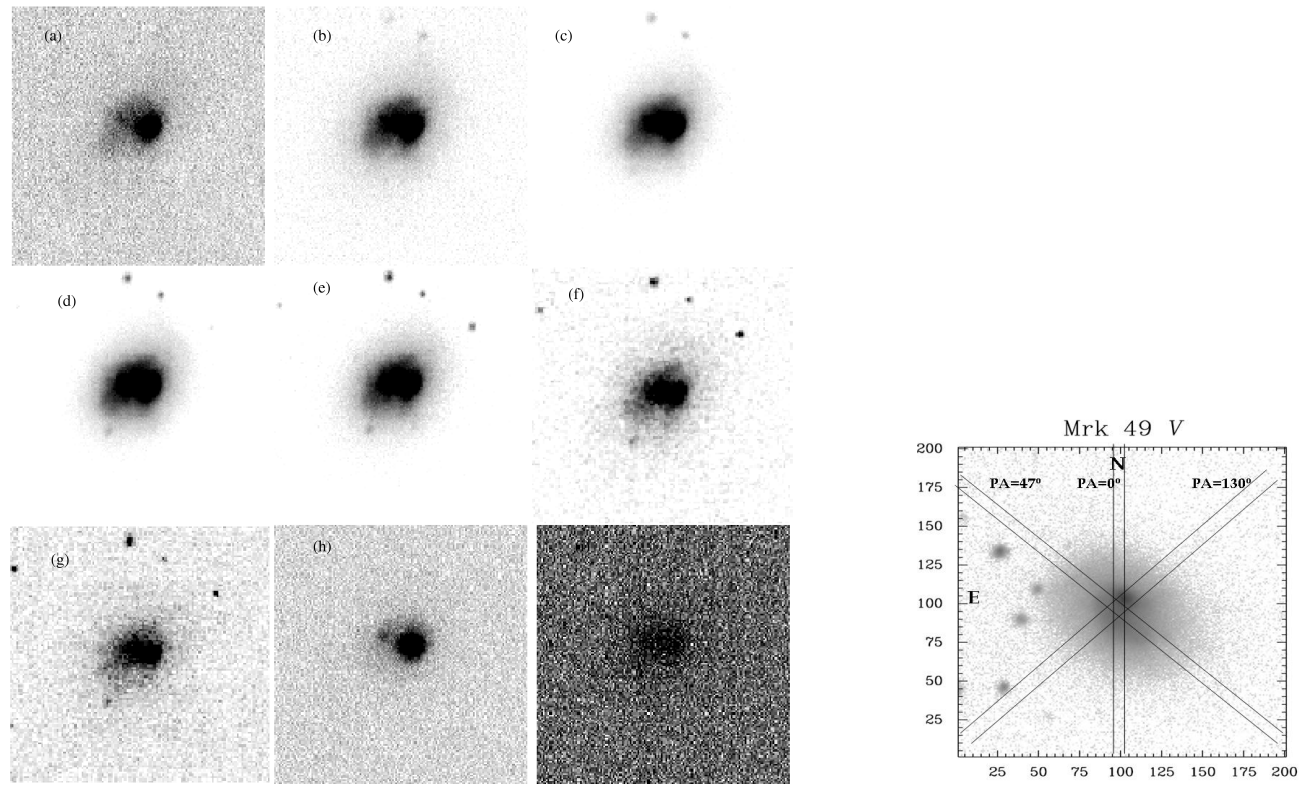

Fig. 1.- Optical and near-infrared images of Mrk 49 (left). East is up and north is to the right. The box size is $1^{\prime} .4 \times$ $1^{\prime} .4$. The filters are (a) $U$, (b) $B$, (c) $V$, (d) $R$, (e) $I$, (f) $J,(\mathrm{~g}) K,(\mathrm{~h}) \mathrm{H}_{\alpha}$, and (i) $\mathrm{H}_{\alpha}$-continuum. The exposure times vary from $480 \mathrm{sec}$ to $1,800 \mathrm{sec}$. Slit positions on the $V$ image(right). Three slit positions, $\mathrm{PA}=0,47,130$ degree are overlaid on the V-band image of Mrk 49. Slit width is $2^{\prime \prime} .7$, and slit length $4^{\prime}$, respectively. The image size is $1^{\prime} .9 \times 1.9$.

TABLE 3.

Spectroscopic OBSERVATions

\begin{tabular}{rrcccc}
\hline \hline & Date & Grating & Wavelength Band & Position Angle $\left(^{\circ}\right)$ & Exposure \\
\hline Apr. 24 & 1993 & 1200 & $\mathrm{H} \alpha(200 \AA)$ & 47 & $1000 \mathrm{sec} \times 2$ \\
26 & 1993 & $"$ & $"$ & 130 & $1000 \mathrm{sec}$ \\
27 & 1993 & $"$ & blue & 0 & $1000 \mathrm{sec}$ \\
Feb. 101994 & 300 & red & 45 & $2000 \mathrm{sec}$ \\
11 & 1994 & & & 45 & $1800 \mathrm{sec}$ \\
\hline
\end{tabular}

was determined from the most probable value counts using the count-to-number histogram. Galaxy images in each filter band were shifted to a common origin, and then one final combined image was obtained using a median filtering method. Photometric calibration was then applied using more than 40 observations of Graham (1982) stars in each passband. In order to calibrate the $\mathrm{H}_{\alpha}$ luminosity, we observed 4 spectroscopic standard stars of Stone \& Baldwin (1983). For the atmospheric extinction coefficients, we adopted the mean values for SSO (Bessell 1994). Landolt's (1992) system was transformed using Bessell's (1990) formulae. The color terms were determined using a response function from the standard stars observation. The surface brightness profiles for each filter were calculated by fitting elliptical isophotes to the sky subtracted images using the STSDAS software package of STScI. The lower intensity values of the image were smoothed with a $3 \times 3$ median point method. For details of the necessary steps and the algorithm of surface photometry, see Drinkwater \& Hardy (1991).

\section{(b) Near-Infrared Imaging}

Near-infrared images were obtained at the $2.2 \mathrm{~m}$ telescope of University of Hawaii equipped with a NICMOS3 IR camera (Hodapp 1992) on 1993 April 5. The camera was set at the $\mathrm{f} / 10$ Cassegrain focus to yield a scale of $0 . \prime 75 /$ pixel and a field view of $3 ! 24 \times 3 ! 24$. During the observation, the seeing was $\sim 11^{\prime \prime} 2$. We used $J$ and $K^{\prime}$ filters; the latter introduced by Wainscoat \& Cowie (1992). The $K^{\prime}$ filter has a $0.35 \mu \mathrm{m}$ FWHM centered at $2.1 \mu \mathrm{m}$, slightly bluer and narrower than the $K$ filter (Bessell 1990). The $K^{\prime}$ filter has an advantage of lower sky brightness compared with the $K$ filter. For 


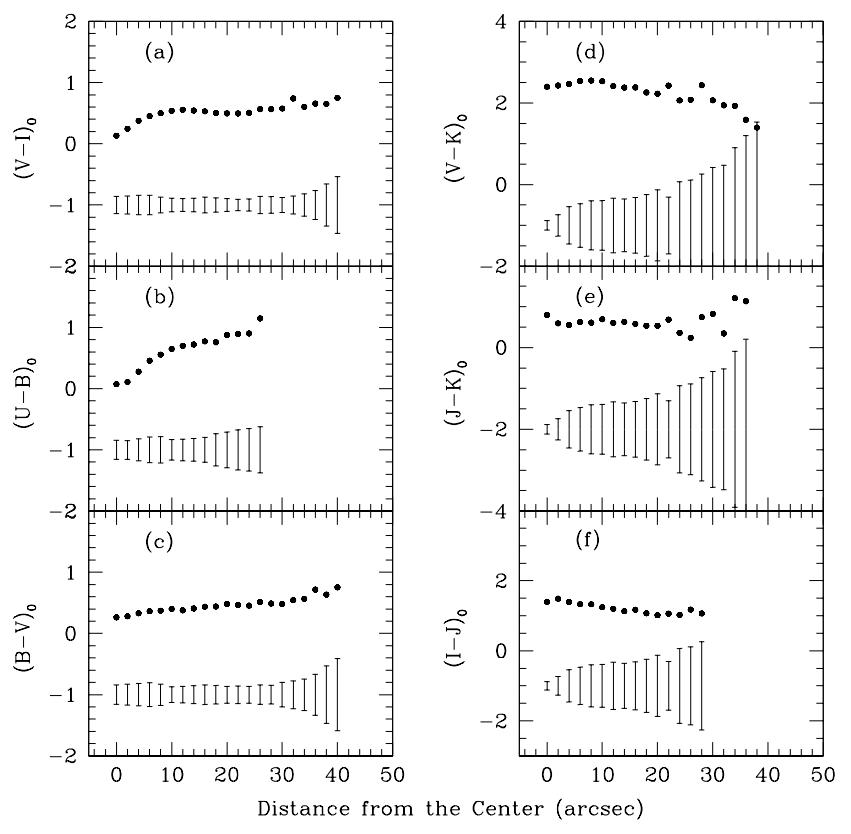

Fig. 2.- Radial distribution of optical and near-infrared color are (a) $V-I$, (b) $U-B$, (c) $B-V$, (d) $V-K,($ e) $J-K$, and (f) $I-J$ respectively. Error bars represent the mean errors of the colors.

the transformation from $K^{\prime}$ to $K$, we used Wainscoat \& Cowie's (1992),

$$
K-K^{\prime}=(0.22 \pm 0.03)(H-K) .
$$

In applying this transformation to Mrk 49, we adopted $H-K=0.25$ from Thuan (1983).

We took 8 galaxy images which were slightly shifted on the array. For each filter, dark frames, and dome flat frames of $60 \mathrm{sec}$ exposure were obtained. Data reduction was done with IRAF. The sky frame was subtracted from the object frame, then which is divided by the flat frame(Joyce 1992). The final set of galaxy images were shifted to a common origin and combined into a final one via median filtering for each filter. Photometric calibration was determined from the observations of 8 UKIRT faint near-infrared standard stars. Surface photometry by ellipse fitting was then applied to the combined image following the same methods as used for the optical CCD images.

\section{(c) Spectroscopy}

Long-slit two-dimensional spectroscopic observations for Mrk 49 were carried out on 1993 April 24 - 27 and on 1994 February 10 - 11 with the Cassegrain spectrograph on the Mount Stromlo Observatory 74 inch telescope equipped with a $385 \times 578$ UV-enhanced CCD camera. In 1993, the 1,200 lines/mm grating, and in 1994, the 300 lines/mm grating were employed, respectively.
The slit width and length were $2^{\prime \prime} .7$ and $4^{\prime}$, respectively for all observations(see Fig. 1(right)). The pointing accuracy is expected to be less than $1^{\prime \prime}$ during our observing runs. For high-resolution spectra (with the 1,200 lines $/ \mathrm{mm}$ grating), we used three different slit position angles of 0,47 , and 130 degrees. Our high resolution spectra cover a wavelength range of $200 \AA$ near $\mathrm{H}_{\alpha}$. A Ne lamp was used for the comparison spectrum. Our low-resolution spectra (with the 300 lines/mm grating) cover the wavelength range $3,600 \AA<\lambda<6,000 \AA$; and $4,700 \AA<\lambda<7,100 \AA$ with a resolution of $4.17 \AA$ /pixel in the blue region and $4.14 \AA /$ pixel in the red region respectively. The slit was positioned along an east-west orientation. A He-Ar lamp was used for the wavelength calibration, and Stone \& Baldwin (1983) standard stars were observed for flux calibration. The data reduction used IRAF.

From the high-resolution spectra, radial velocities were determined using a Gaussian 3 pixels (2'.16) sum fitting to the $\mathrm{H}_{\alpha}$ emission lines along the slit. The wavelength can, therefore, be calculated to heliocentric radial velocities. The estimated errors from night sky lines are $8 \mathrm{~km} / \mathrm{sec}$ for the line flux $I \geq 10^{-13} \mathrm{ergs}$ $\mathrm{sec}^{-1} \mathrm{~cm}^{-2}, 13 \mathrm{~km} / \mathrm{sec}$ for $10^{-13} \geq I \geq 10^{-14} \mathrm{ergs}$ $\mathrm{sec}^{-1} \mathrm{~cm}^{-2}, 18 \mathrm{~km} / \mathrm{sec}$ for $10^{-14} \geq I \geq 10^{-15} \mathrm{ergs}$ $\mathrm{sec}^{-1} \mathrm{~cm}^{-2}$, and $25 \mathrm{~km} / \mathrm{sec}$ for $I \geq 10^{-15} \mathrm{ergs} \mathrm{sec}^{-1}$ $\mathrm{cm}^{-2}$. From the low-resolution spectra, the fluxes of emission lines were measured using Gaussian fitting of the extracted one-dimensional spectra. The flux mea- 
surement errors were estimated as less than $10 \%$ for strong line $\left(I \geq 10^{-13}\right.$ ergs $\left.\mathrm{sec}^{-1} \mathrm{~cm}^{-2}\right)$, and larger than $50 \%$ for weak lines $\left(I \leq 10^{-14} \mathrm{ergs} \mathrm{sec}^{-1} \mathrm{~cm}^{-2}\right)$.

\section{PHOTOMETRIC PROPERTIES}

\section{(a) Morphology}

Fig. 1 shows the optical and near-infrared images of Mrk 49. These images show apparently three components: (1) an off-centered, bright, circular compact object, (2) a bright elliptical region in the center, and (3) a faint diffuse elliptical envelope in the outer region. Because a dust content of Mrk 49 is very low (Parravano 1989), it is hard to exist a dust lane. The $\mathrm{H}_{\alpha}$ image, however, shows that the $\mathrm{H}_{\alpha}$ emission is predominant in the off-centered compact object (see Fig. 1-(h)). This probably suggests that the off-centered compact object have different stellar populations from last two components. These two populations can be called high surface brightness (HSB) massive young stellar population and low surface brightness (LSB) underlying population (Meurer et al. 1992). Our $\mathrm{H}_{\alpha}$ image and the low-dispersion spectra show that star formation is concentrated in the HSB population area, while the LSB population area has a smooth exponential distribution with a nearly constant color (see Fig. 2). Our $\mathrm{H}_{\alpha}$ narrow band image shows that the HSB region is slightly off-centered by $9^{\prime \prime}$ from the isophotal center of the galaxy of the radius $10^{\prime \prime}(0.9 \mathrm{kpc})$ to the north-east direction, while the LSB population is extended by at least $1^{\prime}$ from the center. Optical and near-infrared images show that Mrk 49 appears an elliptical galaxy superimposed on a compact object. From its appearance and the disordered H I velocity field, Lake et al.(1987) suggested the two components could be a result of an interaction. Our observations favor their suggestion. We will discuss this more in the next section.

\section{(b) Luminosities}

We present model free surface photometric parameters in Tab. 4. The magnitudes have been corrected for galactic extinction with $A_{B}=0.02, k$-correction with the radial velocity $V_{r}=1535 \mathrm{~km} / \mathrm{sec}$, and emission line effects (Mackie 1989). The internal absorption is not considered because of its large uncertainty. For the correction of emission lines, we employed Mackie's (1989) formula

$$
\Delta m=-2.5 \log (1+W / P),
$$

where $W$ is a total effective equivalent width of emission lines within the filter band-pass, and $P$ is the FWHM of the filter bandpass. We derived total equivalent widths from our low-dispersion spectra for the $U B V R$ bands. For the $I$ band, we calculated the contribution of [S III] 9096, $9800 \AA$ from the observed intensity of [S II] $6717+6731 \AA$ with an electron temperature of $11,000 \mathrm{~K}$ and an electron number density of

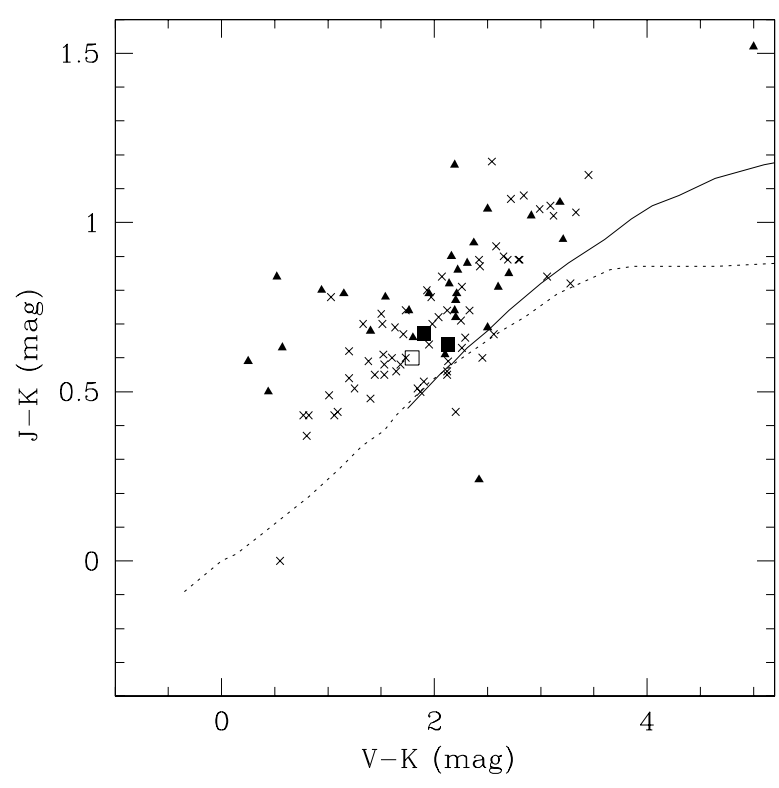

Fig. 3.- Color-color diagram of the optical and nearinfrared images. The cross indicate the LMC \& SMC open clusters(Persson et al. 1983) and the filled triangles BCDs from Thuan(1983). The filled square are the HSB component and the open square the LSB component of Mrk 49. The solid line is for giants and the dotted line for dwarfs (Bessell \& Brett 1988).

100 electrons $\mathrm{cm}^{-3}$ (see Sec. V for detail). For the $J K$ bands, we adopted Thuan's (1983) calculation for Mrk 49. The corrected values are given in Table 5 .

We derived a mean effective surface brightness, $\mu_{e f f}$,

$$
\mu_{\text {eff }}(L)=L_{T}+5 \log \left(r_{\text {eff }}\right)+2,
$$

where $r_{\text {eff }}$ is the effective radius which contains half of the total galaxy light, and $L_{T}$ is the total magnitude in a filter $L$. We obtain a mean effective surface brightness in $B$ of $\mu_{\text {eff }}(\mathrm{B})=21.56 \pm 0.025 \mathrm{mag} \operatorname{arcsec}^{-2}$. The mean effective surface brightness of Mrk 49 is similar to other Virgo BCDs, and 2-3 mag brighter than Virgo dwarf ellipticals (Binggeli \& Cameron 1993). The effective radii in Tab. 4 show larger values for longer wavelengths. This result suggests that the inner region of the galaxy is bluer than the outer part, which is also confirmed by the quantitative study of the light distribution (see below).

In Tab. 6, we compare our results with previously published data. Our $J$ and $K$ images are brighter than Thuan(1983)'s observations because the circular aperture of $8^{\prime \prime}$ was adopted. Our observation agrees well with the others' result within the observational errors. 
TABLE 4.

Model-free Parameters of Surface Photometry

\begin{tabular}{|c|c|c|c|c|c|c|}
\hline \multirow{2}{*}{ Filter } & \multicolumn{3}{|c|}{ Total Magnitude } & \multirow{2}{*}{$\begin{array}{l}\text { Errors } \\
( \pm \mathrm{mag})\end{array}$} & \multirow{2}{*}{$\begin{array}{c}\text { Effective Radius } \\
\text { (arcsec) }\end{array}$} & \multirow{2}{*}{$\begin{array}{c}\text { Radius to } 1.5 \% \text { of sky level } \\
(\operatorname{arcsec})\end{array}$} \\
\hline & observed & corr. $I^{*}$ & corr. I** & & & \\
\hline$U$ & 13.87 & 13.82 & 14.04 & 0.065 & 8.2 & 35.2 \\
\hline$B$ & 14.32 & 14.27 & 14.45 & 0.025 & 11.2 & 51.5 \\
\hline$V$ & 13.80 & 13.77 & 14.17 & 0.015 & 12.3 & 56.6 \\
\hline$R$ & 13.42 & 13.40 & 13.70 & 0.013 & 12.5 & 56.6 \\
\hline$I$ & 13.10 & 13.10 & 13.14 & 0.012 & 13.4 & 56.7 \\
\hline$J$ & 12.72 & 12.73 & 12.76 & 0.038 & 11.3 & 37.2 \\
\hline$K^{\prime}$ & 12.24 & 12.25 & 12.31 & 0.042 & 10.5 & 37.2 \\
\hline$K$ & 12.30 & 12.30 & 12.36 & 0.042 & & \\
\hline
\end{tabular}

* corr. I is corrected galactic extinction and k-correction

** corr. II is corrected galactic extinction, k-correction, and emission line correction

TABLE 5 .

Corrections for Mrk 49. Negative Sign Means Darkening.

\begin{tabular}{cccccr}
\hline \hline Filter & $\begin{array}{c}\text { Galactic extinction } \\
(\mathrm{mag})\end{array}$ & $\begin{array}{c}k \text {-correction } \\
(\mathrm{mag})\end{array}$ & $\begin{array}{c}\text { Emission line effect } \\
(\mathrm{mag})\end{array}$ & \multicolumn{2}{c}{ Total correction } \\
& 0.029 & 0.017 & -0.22 & +0.046 & -0.174 \\
\hline$U$ & 0.020 & 0.027 & -0.18 & +0.047 & -0.133 \\
$B$ & 0.015 & 0.008 & -0.34 & +0.023 & -0.317 \\
$V$ & 0.011 & 0.005 & -0.30 & +0.016 & -0.284 \\
$R$ & 0.003 & 0.001 & $<-0.04$ & +0.004 & $<-0.036$ \\
$I$ & 0.003 & -0.015 & $-0.03^{*}$ & -0.012 & -0.042 \\
$J$ & 0.001 & -0.006 & $-0.06^{*}$ & -0.005 & -0.065 \\
$K^{\prime}$ & 0.001 & -0.006 & $-0.06^{*}$ & -0.005 & -0.065 \\
$K$ & & & &
\end{tabular}

* to be adopted from Thuan(1983)

\section{(c) Color Profiles}

In Tab. 7, we compare the optical and near-infrared colors between the inner HSB region and the outer LSB region. The $J-K$ color of the inner region $\left(R<8^{\prime \prime}\right)$ is 0.1 mag bluer than Thuan's (1983) observation. Optical colors show that the HSB component is bluer than the LSB component. The $V-K$ color and $J-K$ color, however, show the HSB component is redder than the LSB component. This may be due to contributions of the ionized gas emissions. From near-infrared long-slit spectroscopy of other BCDs (Doherty et al. 1995), the contribution of the combined $\mathrm{Br} \gamma$ and He I $2.058 \mu \mathrm{m}$ emissions lines in $K$ band has been estimated as $\sim 5 \%$ from the mean of total $K$ band luminosity for 9 BCDs. On the other hand, Thuan's (1983) estimate was of $4 \%$ contribution in the $K$ band and $3 \%$ contribution in the $J$ band for Mrk 49 within the $8^{\prime \prime}$ aperture. Assuming the upper limit of the contribution of $5 \%$ in $K$ for Mrk 49 , we estimate the contribution of $0.03 \mathrm{mag}$ in $J-K$ and 0.06 mag in $V-K$ color, respectively. Although there may also be other contribution from the ionized gas emissions, it seems clear that this is not the cause of the $J-K, V-K$, and $I-J$ colors of the HSB component being redder than those of the LSB component. Similar result was also shown for NGC 1705 (Quillen et al. 1995). Emission-line corrected $J-K, I-J, V-I$ and $V-K$ colors of the inner-most region $r<5^{\prime \prime}$ show about $0.07,0.3,0.13$ and 0.4 magnitudes redder than the LSB, while $B-V$ and $U-B$ are 0.32 and 0.64 magnitudes bluer than LSB, respectively.

The trends of optical near-infrared colors versus radius are presented in Fig. 2. These colors have been derived from the surface photometry of Mrk 49 through the same apertures. The $(B-V)$ vs $R$ and $(U-B)$ vs $R$ diagrams show bluer inner region, while the $(J-K)$ vs $R$ diagram shows redder inner-most region. The $V-I$, $J-K, I-J$ and $V-K$ colors of LSB are nearly constant, while the $B-V$ color becomes linearly redder with increasing radius. The $V-K$ colors are known to be sensitive to age effect but insensitive to metallicity (Thuan 1983). The constant colors in the $V-I$, $J-K$ and $V-K$ suggest that the LSB has rather homogeneous stellar population.

Fig. 3 shows optical and near-infrared color-color 
TABLE 6.

COMPARISON WITH OTHERS

\begin{tabular}{cccccc}
\hline \hline Filter & This paper* & Huchra $^{1}$ & Thuan $^{2}$ & Gallagher $^{3}$ & NED $^{4}$ \\
\hline U & $13.82 \pm 0.067$ & & $14.32 \pm 0.04$ & $14.56 \pm 0.03$ & $13.68 \pm 0.31$ \\
B & $14.27 \pm 0.025$ & & $14.81 \pm 0.04$ & $14.89 \pm 0.03$ & $14.00 \pm 0.30$ \\
V & $13.77 \pm 0.016$ & $14.64 \pm 0.05$ & $15.42 \pm 0.05$ & $14.54 \pm 0.03$ & $13.51 \pm 0.30$ \\
R & $13.40 \pm 0.013$ & $14.02 \pm 0.03$ & & & \\
I & $13.10 \pm 0.012$ & & $15.06 \pm 0.09$ & & \\
J & $12.73 \pm 0.044$ & & $14.52 \pm 0.11$ & & \\
H & & $14.27 \pm 0.10$ & & 14.21 \\
K & $12.30 \pm 0.062$ & & & & \\
\hline
\end{tabular}

* corr. I values

1 Huchra(1977)

2 Thuan (1983), JHK observations were photoelectric photometry with $8^{\prime \prime}$ circular aperture

3 Gallagher \& Hunter(1986), 30" circular aperture.

4 NASA Extragalactic Database (NED)

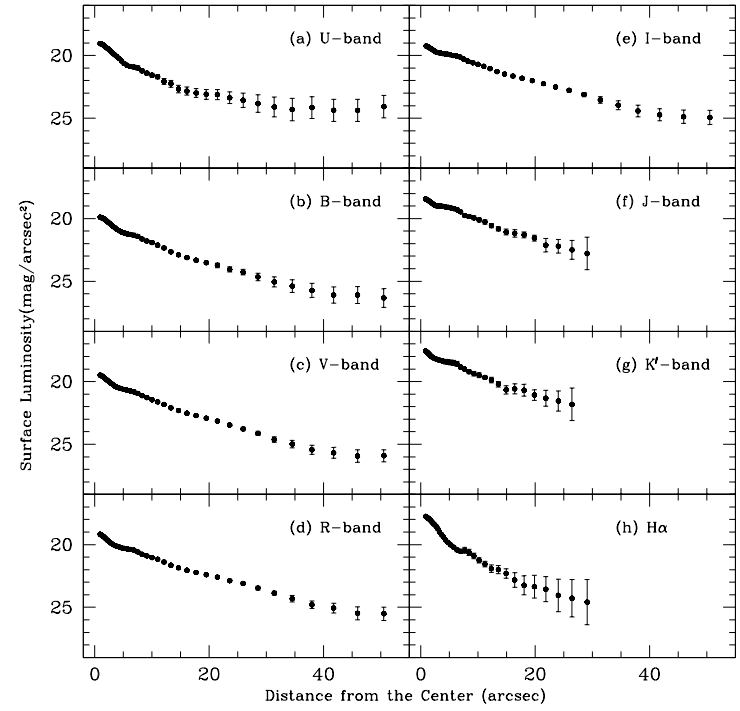

Fig. 4. - Surface brightness profiles to the radial distance in the optical and near infrared images of Mrk 49. From (a) to (h) for $U_{-}, B-, V-, R-, I-, J_{-}, K$-band and $\mathrm{H}_{\alpha}$. Error bars mean the magnitude errors of each points.

diagrams for Mrk 49. The filled squares are the HSB component (both of uncorrected and corrected emission line effects) and the open square is the LSB component. The mean color lines for dwarfs (dotted line) and giants (solid line) are from Bessell and Brett (1988). The filled triangles show the color sample of BCDs from Thuan (1983) and the cross symbols represent open clusters of LMC and SMC from Persson et al. (1983). Quillen et al. (1995) suggested the color of the star cluster in NGC 1705 is similar to that of SWB-type IV-VI

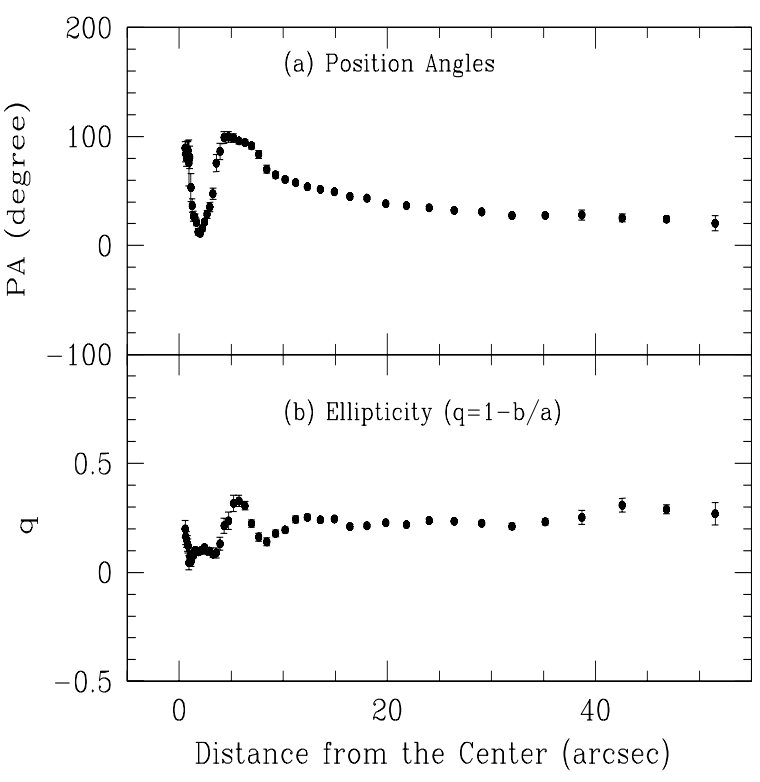

Fig. 5.- (a) Position angle and (b)ellipticity vs. radius. These values were estimated from the $V$-band image of Mrk 49 .

clusters with ages, 1-2 $\times 10^{9}$ years in the Magellanic Clouds. Fig. 3 strongly indicates the existence of supergiant or giant stars in the HSB: the LSB population corresponds to K0 dwarfs or G6 giants group, while the HSB population corresponds to K2-3 giants or dwarfs groups.

\section{(d) Luminosity Pprofiles}

Fig. 4 shows optical and near-infrared surface luminosity profiles of Mrk 49, which can be well described by an exponential disk except for the region of $r \leq 10^{\prime \prime}$. 
The surface luminosity, $\mu(\mathrm{r})$, fitted by an exponential disk can be described by

$$
\mu(r)=\mu_{o}+1.086 \alpha r
$$

where $\mu_{o}$ is the surface luminosity at $r=0$, and $\alpha^{-1}$ is the scalelength. Parameters of the exponential disk are given in Tab. 8. The extrapolated value at the center in the $I$ band is close to the central brightness. So the contribution to the $I$ band luminosity from the gas emission or blue population is minimized.

Fig. 5 shows the distribution of ellipticities and major axis position angles for each ellipse in the $V$ band against its radius, $r=(a b)^{1 / 2}$. Except for the central region $\left(r<10^{\prime \prime}\right)$, the ellipticity and position angle have constant values, 0.25 and 55 degree, respectively. From these values we derived the inclination of the galaxy, $i$, using the following formula,

$$
\cos ^{2} i=\frac{q^{2}+q_{o}^{2}}{1+q_{o}^{2}}
$$

where $q_{o}$ is the mean intrinsic axial ratio; $q=1-$ $b / a$ is an apparent axial ratio. Various $q_{o}$ values have been suggested for BCDs ranging from 0.3 (Gorden \& Gottesmann 1981) to 0.57 (Stavely-Smith et al. 1992). $q_{o}$ is $0.1-0.2$ for spiral galaxies. Staveley-Smith et al.(1992) suggested that the dwarf galaxies would be less flat than disk galaxies because of their relatively small rotational velocities. Our observations for several BCDs, however, show BCDs have rotating disk s whose velocities are comparable to disk galaxies. Thus, we adopt $q_{o}=0.3$. The derived inclination of the galaxy is, $i=39 \pm 5$ degree; and the major axis position angle is $\phi_{o}=55 \pm 5$ degree.

\section{KINEMATICS}

Fig. 6 shows three rotation curves for the slit position angle, $\phi=0,47$, and 130 degree, obtained from our high dispersion spectroscopic observations. The apparent rotation curve at $\phi=47$ shows that the galaxy rotates like a solid body within $r \leq 10^{\prime \prime}$ with the amplitude of $\sim 20 \mathrm{~km} / \mathrm{sec}$. This result confirms the H I observation of Lake et al.(1987). Although the H I velocity field was chaotic, the agreement between the optical and H I observational results suggests that Mrk 49 rotates as a solid body in a plane at the position angle of 55 degree (along the optical major axis) with an amplitude of about $20 \mathrm{~km} / \mathrm{sec}$ in the inner part. Lake et al.(1987) suggested two components of the galaxy, the bright compact component and the faint diffuse elliptical envelope, were an interacting system. Our result also indicates such possibility. From the apparent rotation curve at $\phi=130$, a discontinuity in the velocity rotation curve was found at $r=5^{\prime \prime}$, suggesting two different rotation characteristics; a regular solid body rotation and a constant velocity component for $r \geq 5^{\prime \prime}$ to the east. The relative velocity is about $15 \mathrm{~km} / \mathrm{sec}$.

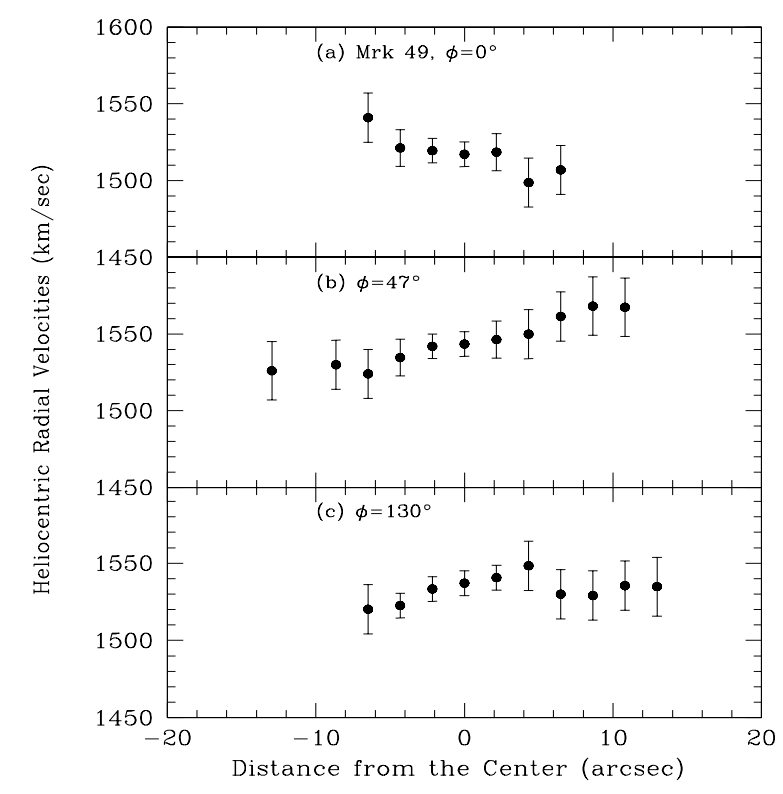

Fig. 6. - Heliocentric radial velocity distributions. (a)the radial variation with the position angle $\phi=0^{\circ}$, (b) for $\phi=$ $47^{\circ}$, and (c) for $\phi=130^{\circ}$.

We measure a heliocentric radial velocity of $1535 \pm 10$ $\mathrm{km} / \mathrm{sec}$. The heliocentric radial velocity agrees well with previous observations within the observational errors; $1523 \mathrm{~km} / \mathrm{sec}$ (Thuan \& Martin 1981), 1516 $\mathrm{km} / \mathrm{sec}$ (Gorden \& Gottesmann 1981), $1518 \mathrm{~km} / \mathrm{sec}$ (Lake \& Schommer 1984), $1582 \mathrm{~km} / \mathrm{sec}$ (Gallagher \& Hunter 1986). Considering the Virgo centric motion (Thuan \& Martin 1981), we derived the distance, $\mathrm{D}=18.2 \mathrm{Mpc}$ with Hubble constant, $\mathrm{H}_{\circ}=75$ $\mathrm{km} \mathrm{sec}^{-1} \mathrm{Mpc}^{-1}$, and the distance modulus, $m-M=$ $31.30 \mathrm{mag}$.

\section{(a) Dynamical Mass}

Although the H I velocity field is chaotic and there exists the possibility of an interaction between two components from the rotation velocity curve analysis along the minor axis, the $\mathrm{H}$ I distribution shows that the gas has strong circular concentration in the central part. Both the optical and infrared images show a smooth elliptical envelope except for the central part; and the galaxy does not show any disturbed feature. Thus, in order to approach simply the kinematics of Mrk 49, we presume the galaxy is rotating along its optical major axis (although there exist local differences in rotating velocities).

Assuming a spherical potential and circular rotation, we follow a similar approach by Bertola et al. (1991). The circular velocity is given as, 
TABLE 7.

Colors of the Inner (HSB) And the Outer Regions (LSB)

\begin{tabular}{|c|c|c|c|c|c|c|c|c|c|c|c|}
\hline \multirow{3}{*}{ Filter } & \multicolumn{6}{|c|}{ HSB (mag) } & \multicolumn{5}{|c|}{$\mathrm{LSB}\left(\mathrm{mag}, \mathrm{r} \geq 10^{\prime \prime}\right)$} \\
\hline & \multicolumn{2}{|c|}{$0-5^{\prime \prime}$} & \multicolumn{2}{|c|}{$5-10^{\prime \prime}$} & \multicolumn{2}{|c|}{$0-10^{\prime \prime}$} & $10-20^{\prime \prime}$ & $20-30^{\prime \prime}$ & $30-40^{\prime \prime}$ & $10-30^{\prime \prime}$ & $10-40^{\prime \prime}$ \\
\hline & $\mathrm{c}_{1}$ & $\mathrm{c}_{2}$ & $\mathrm{c}_{1}$ & $\mathrm{c}_{2}$ & $\mathrm{c}_{1}$ & $\mathrm{c}_{2}$ & $c_{1}$ & $\mathrm{c}_{1}$ & $\mathrm{c}_{1}$ & $\mathrm{c}_{1}$ & $\mathrm{c}_{1}$ \\
\hline$V$ & 15.79 & 16.06 & 15.60 & 15.67 & 14.94 & 15.23 & 14.95 & 15.65 & 16.45 & 14.49 & 14.33 \\
\hline$B-V$ & 0.26 & 0.13 & 0.35 & 0.32 & 0.31 & 0.20 & 0.41 & 0.48 & 0.59 & 0.44 & 0.45 \\
\hline$U-B$ & -0.86 & -0.82 & -0.47 & -0.47 & -0.67 & -0.63 & -0.21 & 0.06 & - & -0.13 & -0.13 \\
\hline$U-V$ & -0.60 & -0.69 & -0.12 & -0.15 & -0.36 & -0.43 & 0.20 & 0.54 & - & 0.31 & 0.35 \\
\hline$V-R$ & 0.34 & 0.37 & 0.38 & 0.39 & 0.36 & 0.37 & 0.38 & 0.40 & 0.40 & 0.38 & 0.39 \\
\hline$V-I$ & 0.39 & 0.63 & 0.63 & 0.71 & 0.54 & 0.79 & 0.71 & 0.77 & 0.75 & 0.73 & 0.73 \\
\hline$V-K$ & 1.87 & 2.09 & 1.91 & 1.97 & 1.90 & 2.13 & 1.84 & 1.70 & - & 1.79 & 1.74 \\
\hline$J-K$ & 0.75 & 0.72 & 0.61 & 0.61 & 0.67 & 0.64 & 0.62 & 0.57 & - & 0.60 & 0.62 \\
\hline$I-J$ & 0.73 & 0.74 & 0.65 & 0.65 & 0.69 & 0.70 & 0.51 & 0.36 & - & 0.46 & 0.39 \\
\hline$B-J$ & 1.38 & 1.50 & 1.65 & 1.68 & 1.54 & 1.69 & 1.63 & 1.61 & - & 1.63 & 1.57 \\
\hline
\end{tabular}

TABLE 8.

Model Dependent Parameter of MrK 49

\begin{tabular}{ccccc}
\hline \hline \multirow{2}{*}{ Filter } & \multicolumn{2}{c}{ Central Mag. $\left(\mathrm{mag} / \square^{\prime \prime}\right)$} & \multicolumn{2}{c}{ Scale Length } \\
& Actual & Extrapolated & (arcsec) & $(\mathrm{kpc})$ \\
\hline$U$ & $18.96 \pm 0.06$ & $22.59 \pm 0.10$ & 6.7 & 0.60 \\
$B$ & $19.96 \pm 0.03$ & $20.44 \pm 0.07$ & 7.1 & 0.64 \\
$V$ & $19.57 \pm 0.01$ & $20.42 \pm 0.05$ & 8.7 & 0.78 \\
$R$ & $19.17 \pm 0.01$ & $20.16 \pm 0.06$ & 9.3 & 0.83 \\
$I$ & $19.26 \pm 0.01$ & $19.31 \pm 0.05$ & 7.3 & 0.65 \\
$J$ & $18.06 \pm 0.04$ & $18.52 \pm 0.04$ & 6.6 & 0.59 \\
$K$ & $17.37 \pm 0.04$ & $18.56 \pm 0.05$ & 5.9 & 0.53 \\
\hline
\end{tabular}

$$
v_{c}(r)=\frac{A r}{\left(r^{2}+c_{o}^{2}\right)^{p / 2}},
$$

where $A, c_{o}$, and $p$ are parameters which described below can be determined simultaneously by fitting observations.

For $r \leq 10^{\prime \prime}, v_{c}(r) \propto r . A=2.9 \pm 0.1 \mathrm{~km} \mathrm{sec}^{-1}$ $\operatorname{arcsec}^{-1}$ can be determined from our rotation curves. From $\mathrm{Eq}(6)$, the mass distribution $M(r)$ is given as,

$$
M(r)=\frac{1}{G} r_{V_{c}}^{2} r=\frac{A^{2} r^{3}}{G\left(r^{2}+c_{0}^{2}\right)^{p}}
$$

where $\mathrm{G}$ is the gravitational constant. For $r \leq 10^{\prime \prime}$, the dynamical mass of Mrk 49, $M\left(r=10^{\prime \prime}\right)=\overline{A^{2}} r^{3}$ $\mathrm{G}=(1.7 \pm 0.6) \times 10^{8} \mathrm{M}_{\odot}$. The mass-to-light ratio, $M / L_{I} \cong 1.0$ in the $I$ band and $M / L_{B} \cong 0.5$ in the $B$ band. The mass-to-light ratios of Mrk 49 are smaller than spiral galaxies or dwarf galaxies. Relatively small mass-to-light ratios than other galaxies would be due to existence of hot massive stars in the central region of Mrk 49.

\section{(b) Gas Mass}

The mass of H II gas within $r=10^{\prime \prime}$ can be estimated from the $\mathrm{H}_{\beta}$ luminosity of Mrk 49. The mass of H II, $M_{H I I}$ (Meurer et al. 1992) is,

$$
M_{H I I}=\frac{(1+Y) L_{H_{\beta}} m_{p}}{\alpha_{H_{\beta}}^{e f f}\left(T_{e}\right) h \nu_{H_{\beta}}\left[N_{e}\right]}
$$

where $Y$ is the mass fraction of helium(adopted 0.23 from our low dispersion spectroscopy); and $L_{H_{3}}$ is the $H_{\beta}$ luminosity, $m_{p}$ is the mass of proton, 1.6725 $\times 10^{-24} \mathrm{~g}$, and $\alpha_{H_{\beta}}^{e f f}\left(T_{e}\right)$ is the $\mathrm{H}_{\beta}$ effective recombination coefficient at the electron temperature, $T_{e}$ which is derived from the intensity ratio [O $\mathrm{III}] \lambda \lambda$ $(4959+5007) / 4363$ (Osterbrock(1975); McCall(1984)). The Planck constant $\mathrm{h}=6.626 \times 10^{-27} \mathrm{cgs}, \lambda_{H_{\beta}}$ is $\mathrm{H}_{\beta}$ the wavelength, and $\left[N_{e}\right]$ is the mean electron number density. We adopt $T_{e}=11,000 \mathrm{~K}$ and $\alpha_{H_{\beta}}^{e f f}\left(T_{e}\right)=2.79$ $\times 10^{-14} \mathrm{cgs}$ (McCall 1984). The mean electron density $\left[N_{e}\right]$ is highly uncertain. We tried to derive from the 
ratio of $[\mathrm{S} \mathrm{II}] \lambda 6717 / \lambda 6731$ (Osterbrock(1975); McCall(1984)), but the ratio is larger than 1.49 for Mrk 49. Thus the electron number density is the limit of electron density, $N_{e} \leq 100$ electrons $\mathrm{cm}^{-3}$ (Osterbrock 1975). The lower limit of the electron density can be derived by modifying Eq. (8),

$$
\left[N_{e}\right]_{\min }^{2}=\frac{(1+Y) L_{H_{\beta}} \lambda_{H_{\beta}}}{\alpha_{H_{\beta}}^{e f f}\left(T_{e}\right) h c V}
$$

where c is the speed of light, and $V$ is the volume containing with the radius $\leq 10^{\prime \prime}$. We adopt $L_{\beta}=1.7 \times$ $10^{41} \mathrm{erg} \mathrm{sec}^{-1} \mathrm{~cm}^{-2}$ from our low dispersion spectroscopic observation. Assuming a volume filling factor is $1,4.7 \leq\left[N_{e}\right]<100$ electrons $\mathrm{cm}^{-3}$. Using the aforementioned relation, Eq. (8) becomes

$$
M_{H I I}=\frac{1.54 \times 10^{9}}{\left[N_{e}\right]} M_{\odot}
$$

Thus, the H II mass within $\mathrm{r}=10^{\prime \prime}$ is $1.5 \times 10^{7} \leq$ $M_{H I I} \leq 3.3 \times 10^{8} M_{\odot}$. We adopt $M_{H I I}=1 \times 10^{8} \mathrm{M}_{\odot}$ which is somewhat in the middle.

Crudely estimated neutral hydrogen mass based on the H I observation of the Lake et al. (1987) within $r=10^{\prime \prime}, M_{H I}=3 \times 10^{7} M_{\odot}$. Correcting for the helium content, we finally get a total mass of interstellar medium (ISM) within $r=10^{\prime \prime}$ for Mrk 49, $M_{\text {gas }}\left(r<10^{\prime \prime}\right)(1+Y)\left(M_{H I}+M_{H I I}\right)=1.6 \times 10^{8} M_{\odot}$. The resulting mass seems to be overestimated possibly because of stellar components, appearing in the $I$ band luminosity profile. The lower limit of gas mass is $5.5 \times$ $10^{7} M_{\odot}$. If we adopt equal splitting between ISM and stellar component, the upper limit of the gas mass will be $\sim 1 \times 10^{8} \mathrm{M}_{\odot}$.

Various results on the Mrk 49 total H I mass have been derived from $21 \mathrm{~cm}$ radio observations in the literatures: $1.9 \times 10^{8} \mathrm{M}_{\odot}$ by Thuan \& Martin (1981), $1.74 \times 10^{8} \mathrm{M}_{\odot}$ by Gordon \& Gottesman(1981), and $4.37 \times 10^{8} \mathrm{M}_{\odot}$ by Lake \& Schommer (1984). We use a total mass of $\mathrm{H} \mathrm{I}, M_{H I}(\mathrm{t})=2 \times 10^{8} \mathrm{M}_{\odot}$, and a total mass of gas, $M_{\text {gas }}(\mathrm{t}) \simeq 4 \times 10^{8} \mathrm{M}_{\odot}$. The dust mass of Mrk 49 was estimated from IRAS flux as $4 \times$ $10^{4} \mathrm{M}_{\odot}$ (Parravano 1989). From the relation between a dust mass and a $\mathrm{H}_{2}$ mass of dwarf irregular galaxies (Hunter et al. 1989), $M_{H I I}=250 M_{d u s t}$, we estimated the molecular hydrogen mass, $M_{H I I} \simeq 1 \times 10^{7} \mathrm{M}_{\odot}$. A dust-to-gas mass ratio of $\operatorname{Mrk} 49, M_{d u s t} / M_{\text {gas }} \cong$ $1 \times 10^{-4}$, which is a typical dust-to-gas mass ratio of BCDs.

\section{(c) Total mass}

The H I gas appears to extend out to $1^{\prime} .5$, while our CCD observations show about $1^{\prime}$ at $1 \%$ sky level. In the case of a spherical isothermal galaxy, a total mass of the system (Stavely-Smith et al. 1992), $M_{T}$, is

$$
\frac{M_{T}}{M_{\odot}}=3.3 \times 10^{4} \alpha_{H} D\left(3 \sigma^{2}+\operatorname{Vrot}^{2}\right)
$$

where $\alpha_{H}$ is Holmberg radius $\left(0^{\prime} .65\right.$ for Mrk 49); $D$ the distance, $18.2 \mathrm{Mpc} ; V_{\text {rot }}$ the rotational velocity at the Holmberg radius(adopted $25 \mathrm{~km} / \mathrm{sec}$ ); and $\sigma$ an isotropic velocity dispersion was $10 \mathrm{~km} / \mathrm{sec}$, which is the mean value of dwarf galaxies (Shostak \& van der Kruit 1984). From Eq. (11), we obtain the total mass of $3.6 \times 10^{8} \mathrm{M}_{\odot}$. This value is smaller than estimated total gas mass of Mrk 49.

Assuming the power-law density distribution, the mass distribution (Binney \& Tremaine 1987), $M(r)$,

$$
\rho(r)=\rho_{o}\left(r_{o} / r\right)^{3-\alpha} .
$$

where $r$ is a radius, $\alpha$ a parameter, $\rho(\mathrm{r})$ the density distribution, and $\rho_{o}$ a central density. Fig. 6 shows the rotational velocity which seems to be a constant for $\mathrm{R}$ $\geq 10^{\prime \prime}$. If $v(r)=$ constant in the region $r \gg 1, \alpha \cong 2$ as in a spiral galaxy. In such a case, we find $\mathrm{M}_{t o t}\left(\mathrm{r}=60^{\prime \prime}\right)$ $=7 \times 10^{9} \mathrm{M}_{\odot}$.

The other method for the total mass estimation was presented by Bucherhorn(1992). For a large sample of spiral galaxies, Bucherhorn (1992) found the ratio of the mass to $I$ band luminosity $M / L_{I}=$ constant. Freeman (1992) suggested the constant value of 4 for the spiral galaxies; $>12$ for dwarf galaxies of $\mathrm{M}_{B} \leq-12$, 3 for $-14.5 \leq \mathrm{M}_{B} \leq-12$; and 5 for dwarf spheroidals. Adopting $\bar{M} / L_{I}=12$, the total mass is $M_{t o t}=8 \times$ $10^{9} \mathrm{M}_{\odot}$. The total mass of the galaxy is very uncertain. The upper and lower limit to the total mass $M_{t o t}$ can be found from the total gas mass with the power density assumption. Therefore, the total mass $M_{t o t}$ is $4 \times 10^{8} \mathrm{M}_{\odot} \leq M_{t o t} \leq 7 \times 10^{9} \mathrm{M}_{\odot}$.

\section{ABUNDANCES}

Fig. 7 shows low dispersion spectra for Mrk 49. The spectra show typical sharp narrow emission lines of BCDs. Emission line ratios were derived from the extracted one-dimensional spectra. These are given in Tab. 9. The reddening correction has been applied using the following equation,

$$
\log F_{c o r}=\log F_{o b s}+C\left(H_{\beta}\right) f(\lambda)
$$

where $F_{c o r}$ is corrected flux, and $F_{o b s}$ is observed flux of emission line, $C\left(\mathrm{H}_{\beta}\right)$ is reddening coefficient in $\mathrm{H}_{\beta}$, derived from a comparison of observed Balmer decrements with calculated values for $T_{e}=10,000 \mathrm{~K}$ and $N_{e}=100$ (McCall 1984), and $f(\lambda)$ is the reddening function (Seaton 1979). We obtained $C\left(\mathrm{H}_{\beta}\right)=0.28$.

The elemental abundances can be estimated empirically from observed emission line intensities and can be used to $T_{e}$ and $N_{e}$ (Dinerstein \& Shields 1986). We adopt atomic constants from Mendoza (1983) and McCall (1984). We derived an electron temperature $T_{e}=$ $11,000 \pm 1000 \mathrm{~K}$ from $[\mathrm{O}$ III $] \lambda \lambda(4959+5007) / 4363$. 

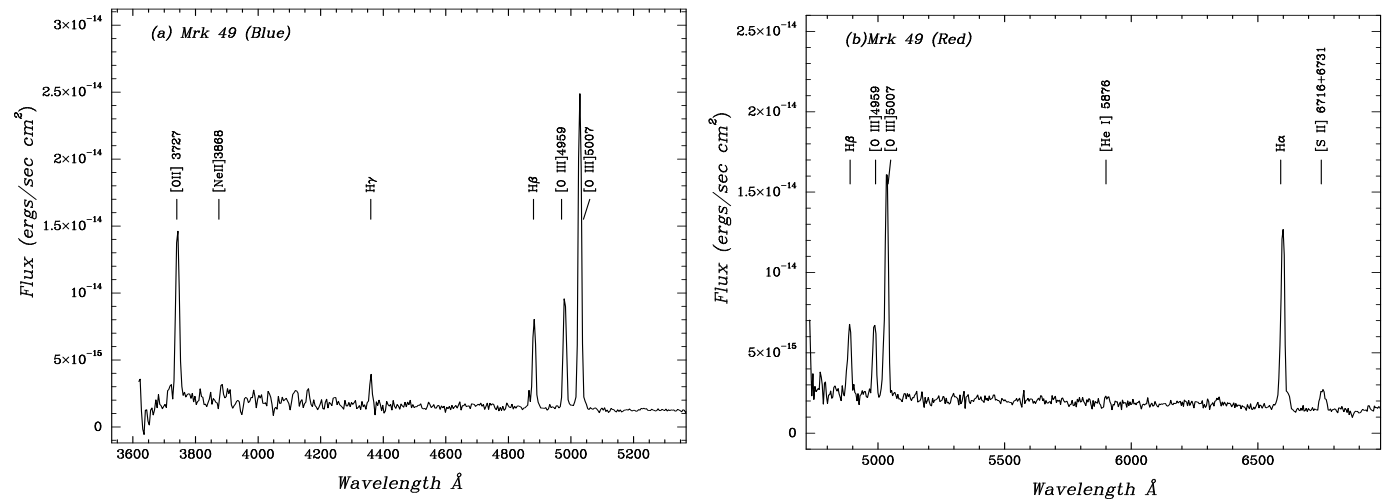

Fig. 7. - Spectroscopic observation of the Mrk 49 in (a) blue region and nd (b) the red region.

We chose an electron number density $N_{e}=100$ electron $\mathrm{cm}^{-3}$, because [S II] $\lambda \lambda 6717 / 6731$ line ratio is larger than the value of the lower density limit, 1.49 . Since [O III] $\lambda 4363$ is week and probably contaminated by night sky lines, the electron temperature is a large error: 0.2 dex error in oxygen abundance determination. The derived oxygen abundance agrees well with previous published data(Shi et al. 2005, Vilchez \& Iglesias-Paramo 2003). Derived elemental abundances are given in Tab. 10, along with other published data of BCDs and H II regions (Melnick et al. 1992). The helium abundance of Mrk 49 is similar to those of the other BCDs. Thuan (1983) suggested a linear relationship between $(J-K)$ color and its oxygen abundance for BCDs as in the $(J-K)$ color-metallicity relationship for globular clusters. We found Mrk 49 agrees well with this relationship.

\section{DISCUSSION AND SUMMARY}

\section{(a) General Morphology and Comparison to Other Galaxies}

Our optical and near infra-red observations show Mrk 49 has two components: the off-centered bright object with a radius of $10^{\prime \prime}$ and the outer faint diffuse elliptical envelope out to about $1^{\prime}$ from the center. The $\mathrm{H}_{\alpha}$ morphology of Mrk 49 from a narrow-band observation shows that the off-centered bright component displays predominantly $\mathrm{H}_{\alpha}$ emissions and massive star-forming spectral futures.

Our long-slit spectroscopy at the position angle of 0 and 130 degree shows a discontinuity in the velocity curve at $\mathrm{r}=5$ arcsec. This result suggests two components, the bright compact (HSB) and the faint diffuse elliptical envelope (LSB), are counter-rotating. The velocity difference between two components from our long-slit spectroscopy are well agreed with the result from HI image observation by Lake et al (1987). The evidences of merger remnants are suggested count rotating cores, shell morphology, HI tails and disturbed HI kinematics after the fast relaxation of the stellar components within 1Gyr (Schweizer \& Seitzer 1992, Mihos \& Hernquist 1994, Bekki 2008). Recent studies show that at least $70 \%$ of BCDs are mergers or interactions (Sung 2000, Pustilnik et al 2001, Bekki 2008). But however, until now, only few BCDs are known as post-mergers; He 2-10 (Kobulnicky et al. 1995), Mrk 996 (Thuan et al. 1996), and Tololo 3 (Sung et al. 2002). Mrk 49 has similar observational characteristics to He 2-10. Thus, from the observational clues, we suggest that Mrk 49 should be a post merger.

\section{(b) Origin of Near-Infrared Light}

One possible explanation for the redder near-infrared colors in the HSB region is the existence of red supergiant stars in the HSB region (Campbell \& Terlevich 1984; Vanzi et al.1996). Another possible answer is Joy \& Lester (1988)'s result for near-infrared line and continuum emission from BCD II Zw 40. They suggested nebular recombination emission and photoshperic radiation from young blue stars would produce most of the near-infrared continuum emission in the central $6^{\prime \prime}$ of II Zw 40. The surface luminosity profiles of Mrk 49 showed in Fig 4 suggest that radiation from young blue stars is not major of the near-infrared light in the HSB region of Mrk 49. Another possible answer may be found from Thuan (1983), Hunter \& Gallagher(1985), and Deharveng et al.(1994)'s suggestion. Thuan (1983) suggested that the near-infrared flux of BCDs originates from an old population of $\mathrm{K}$ and $\mathrm{M}$ giants, and Hunter \& Gallagher (1985) argued young population is not a major contribution to the near-infrared light. Deharveng et al.(1994) presented Hubble Space Telescope(HST) observations for the central cluster of I 
TABLE 9.

Reddening Corrected Emission Line Ratio to H $\beta$

\begin{tabular}{ccccc}
\hline \hline ion & $\lambda$ & $\mathrm{f}(\lambda)$ & Fobs & Fcor \\
\hline$[\mathrm{O} \mathrm{II}]$ & 3727 & 0.255 & 2.157 & 2.542 \\
{$[\mathrm{Ne} \mathrm{III}]$} & 3869 & 0.235 & 0.170 & 0.198 \\
$\mathrm{H} \delta$ & 4102 & 0.172 & 0.210 & 0.230 \\
$\mathrm{H} \gamma$ & 4340 & 0.125 & 0.316 & 0.450 \\
{$[\mathrm{O} \mathrm{III}]$} & 4363 & 0.124 & 0.031 & 0.033 \\
$\mathrm{H} \beta$ & 4861 & 0.000 & 1.000 & 1.000 \\
{$[\mathrm{O} \mathrm{III}]$} & 4959 & -0.022 & 1.281 & 1.263 \\
{$[\mathrm{O} \mathrm{III}]$} & 5007 & -0.033 & 3.844 & 3.763 \\
$\mathrm{He} \mathrm{I}$ & 5876 & -0.208 & 0.127 & 0.111 \\
$\mathrm{H} \alpha$ & 6563 & -0.322 & 3.289 & 2.672 \\
{$[\mathrm{~N} \mathrm{II}]$} & 6584 & -0.325 & 0.127 & 0.111 \\
{$[\mathrm{~S} \mathrm{II}]$} & 6717 & -0.343 & 0.286 & 0.229 \\
{$[\mathrm{~S} \mathrm{II}]$} & 6731 & -0.344 & 0.102 & 0.082 \\
\hline $\mathrm{F}(\mathrm{H} \beta)=9.7 \times 10^{-13} \mathrm{erg} \mathrm{sec}^{-1} \mathrm{~cm}^{-2}$
\end{tabular}

TABLE 10

Total Abundances. Unit is $12+$ LOG $(\mathrm{X} / \mathrm{H})$

\begin{tabular}{cccccc}
\hline \hline Galaxy & $\mathrm{He}$ & $\mathrm{O}$ & $\mathrm{N}$ & $\mathrm{S}$ & $\mathrm{Ne}$ \\
\hline Mrk 49* & $10.91 \pm 0.2$ & $8.21 \pm 0.1$ & $6.97 \pm 0.3$ & $6.97 \pm 0.3$ & $7.23 \pm 0.15$ \\
E105-IG11** & 10.84 & 7.76 & 6.72 & 7.47 & 6.99 \\
Sun & 10.93 & 8.82 & 7.68 & 7.30 & 7.92 \\
Orion & 11.00 & 8.79 & 7.63 & 7.50 & 7.90 \\
30 Dor & 10.92 & 8.38 & 6.99 & 7.41 & 7.74 \\
NGC 604 & 10.92 & 8.51 & 7.35 & 6.95 & 7.53 \\
I Zw 18 & 10.71 & 7.18 & 5.60 & 6.98 & 6.55 \\
NGC5471 & 10.76 & 8.13 & 6.51 & 6.93 & \\
\hline
\end{tabular}

* this paper

** Sung et al.(1996)

Zw 36 in UV and optical band. They reported the integrated UV-optical color was redder than what we would expect from burst of star formation (the age $\leq$ $12 \mathrm{Myrs}$ ); and they suggested the presence of an older and unresolved underlying population in the galaxy. They argued the contribution of a few red supergiants to the integrated stellar population remained marginal. In the case of Mrk 49, it is possible to have the older underlying population in the HSB: The older underlying population is possibly the population of the LSB. In case when the underlying population has the same colors of the LSB, one cannot explain why the nearinfrared colors for the inner most HSB region is reddish. Hence, a young cluster containing evolved cool stars of luminosity classes Ia and $\mathrm{Ib}$ are possibly co-existent with young and less massive stars as well as the older underlying population. The recent HST observations showed a number of yellow and red supergiants in superclusters of NGC 1569 and NGC1705 (O'Connell et al. 1994), and I Zw 18(Hunter \& Thronson 1995). The M1 Ia supergiants with $\mathrm{M}_{I} \approx-10$ were also found in NGC 1569 and NGC 1705.

The underlying populations (LSB) of BCDs are significantly redder than dIs, but bluer than dEs or normal spirals and ellipticals (Sung 2000, Noeke et al. 2005, Papaderos et al. 2006). Our photometric result for the LSB component of Mrk 49 shows similar opticalnear infrared colors to BCDs by Noeske et al (2005). One of possible explanation for the bluer colors than $\mathrm{dEs}$ or normal galaxies is the merging scenario. Merging process is rather fast, and the stellar component is fast relaxation within 1Gyr (Mihos \& Hernquist 1994, Bekki 2008). And, thus, new born young population is possibly mixed with the older underlying population. Therefor, most of BCDs have bluer disks than dEs or normal galaxies due to the younger population, and redder than dIrr due to young bright supergiants or giants. 
The age can be estimated by investigating the onset of star formation model on the $(U-B)-(V-K)$ color plane of Struck-Marcell \& Tinsley(1978). The HSB population appears to be younger than $2 \times 10^{7}$ years, while the LSB population seems to be older than $1 \times 10^{9}$ years. All massive $\operatorname{stars}\left(>15 \mathrm{M}_{\odot}\right)$ may have evolved into the giant or red supergiant stage.

\section{(c) Dark Matter Content}

The dynamical mass determined from rotation curves with $r \leq 10^{\prime \prime}$ is $1.7 \times 10^{8} \mathrm{M}_{\odot}$, and the mass of gas is $8.6 \times 10^{7} \mathrm{M}_{\odot}$. The mass-to-light ratio for the central region of Mrk 49 is estimated as $M / L_{I} \cong 1.0$ in $I$ band and $M / L_{B} \cong 0.5$ in $B$ band. It appears that the local dark matter content is not very high for the optical core of Mrk 49 like NGC 2915 (Meurer et al. 1994). Since the total mass is uncertain, the mass-to-light ratio will be very uncertain. The total gas mass was adopted as $M_{\text {gas }}($ tot $)=4 \times 10^{8} \mathrm{M}_{\odot}$. The recent study for He 2-10 was presented for the molecular mass comparing to the atomic mass (Kobulnicky et al. 1995). Considering H I and $\mathrm{H}$ II with $\mathrm{H}_{2}$ mass, the lower limit of total mass for Mrk 49 is $6 \times 10^{8} \mathrm{M}_{\odot}$. If we consider the turbulence mass (Thuan \& Martin 1981), then the mass limit $\cong$ $7 \times 10^{8} \mathrm{M}_{\odot}$. The total mass is $7 \times 10^{8} \geq M_{t o t} \geq 7$ $\times 10^{9} \mathrm{M}_{\odot}$. Therefore the mass-to-light ratios are 0.5 $\geq M / L_{I} \geq 5,0.9 \geq M / L_{B} \geq 9$. The mass-to-light ratio is not different from that of NGC 1705 (Meurer et al. 1992). The mass-to-light ratio in I band for Mrk 49 $\left(M_{B}=-17.0\right), M / L_{I}$, seems to be slightly higher than dwarf galaxies with $-14.5 \geq M_{B} \geq-12$. The upper limit of the dark matter to visible matter ratio is less than 5. In Tab. 11, we compare the our results with NGC 1705 (Meurer et al. 1992).

\section{(d) Summary}

We summarize our results as follows:

1. The optical image of Mrk 49 shows a bright compact object in the center and the faint diffuse elliptical envelope.

2. The size of $\mathrm{H}$ II region is about to $12^{\prime \prime}(1 \mathrm{kpc})$ and is located at the $10^{\prime \prime}$ distance away from the underlying low surface brightness regular shape component. The contour map of $\mathrm{H}_{\alpha}$ image shows a bright compact object has a circular feature, while broad-band images show a bar-like feature.

3. The surface luminosity distribution of Mrk 49 indicates the existence of the exponential disc beyond the central $10^{\prime \prime}$ region. The total apparent blue magnitude is $\mathrm{B}_{T}=14.32 \pm 0.025 \mathrm{mag}$., and the mean effective surface brightness $\mu_{\text {eff }}=21.57 \mathrm{mag} \operatorname{arcsec}^{-2}$. This value is a typical case of the blue compact galaxy in the Virgo Cluster.

4. The ellipticity distribution along the radius shows fluctuation in the central region, but converges to a constant value beyond $20^{\prime \prime}$ in the radial distance. The axial ratio at $R_{26}$ is $0.25 \pm 0.03$ and the position angle is $145^{\circ} \pm 5$.

5. The heliocentric radial velocity of Mrk 49 of 1535 $\pm 10 \mathrm{~km} / \mathrm{sec}$ was derived from the spectroscopic observations.

6. The rotation velocity curve along the major axis shows the rigid rotation with a maximum amplitude of $20 \mathrm{~km} / \mathrm{sec}$ until $\mathrm{r} \leq 10^{\prime \prime}$. The mass of Mrk 49 inside the $\mathrm{r}=10^{\prime \prime}$ was estimated as $1.7 \times 10^{8} \mathrm{M}_{\odot}$. In this area the total mass of $\mathrm{H}$ II region was $1.7 \times 10^{8} \mathrm{M}_{\odot}$ which agrees well with the estimate from rotation curve.

7. Assuming the mass to luminosity ratio in I band image as 4, we estimate the total mass of Mrk 49 as 2.8 $\times 10^{9} \mathrm{M}_{\odot}$. Under the assumption of the power-law density distribution and rotation velocity beyond $\mathrm{r}=$ $10^{\prime \prime}$ fixed as $25 \mathrm{~km} / \mathrm{sec}$, we derive the total mass of Mrk 49 as $6.9 \times 10^{9} \mathrm{M}_{\odot}$ within $\mathrm{r}=60^{\prime \prime}$. From these values, the total mass of Mrk 49 appears to be in the range of 3 to $6 \times 10^{9} \mathrm{M}_{\odot}$.

8. The electron temperature and the electron density were derived as $11000 \pm 800 \mathrm{~K}$ and less than 100 electron $\mathrm{cm}^{-3}$, respectively, from the low dispersion spectrum. The oxygen abundance $12+\log (\mathrm{O} / \mathrm{H})$ is $8.21 \pm 0.1$ which is larger than the average abundance of a typical blue compact galaxy. The helium abundance $12+\log (\mathrm{He} / \mathrm{H})$ is estimated as $10.91 \pm 0.2$, similar to that of solar value.

We are indebted to Professor K. C. Freeman for his various helpful advice. We appreciate assistance of staffs at Mount Stromlo and Siding Spring Observatories during our observing runs. YIB acknowledges the support of Korea Research Foundation (KRF-2007C00020).

\section{REFERENCES}

Bekki, K., 2008, Formation of blue compact dwarf galaxies from merging and interacting gas-rich dwarfs, MNRAS, 388, 10

Bertola, F., Bettoni, D. A., Danziger, J., Sadler, E., Sparke, L., \& de Zeeuw, T., 1991, Testing the gravitational field in elliptical galaxies - NGC 5077, ApJ, 373,369

Bessell, M. S., 1990, UBVRI passbands, PASP, 102, 1181

Bessell, M. S. \& Brett, J. M., 1988, JHKLM photometry - Standard systems, passbands, and intrinsic colors, PASP, 100. 1134

Binggeli B. \& Cameron, L. M., 1993, Dwarf galaxies in the Virgo cluster. II - Photometric techniques and basic dat, A\&AS, 98, 297

Bingelli B., Popescu C. C., \& Tammann G. A., 1993, The kinematics of the Virgo cluster revisited, A\&AS, 98,275

Binney, J. J. \& Tremaine, S., 1987, Galactic Dynamics, Princeton University Press, 41 
TABLE 11.

Comparison to Other Galaxies

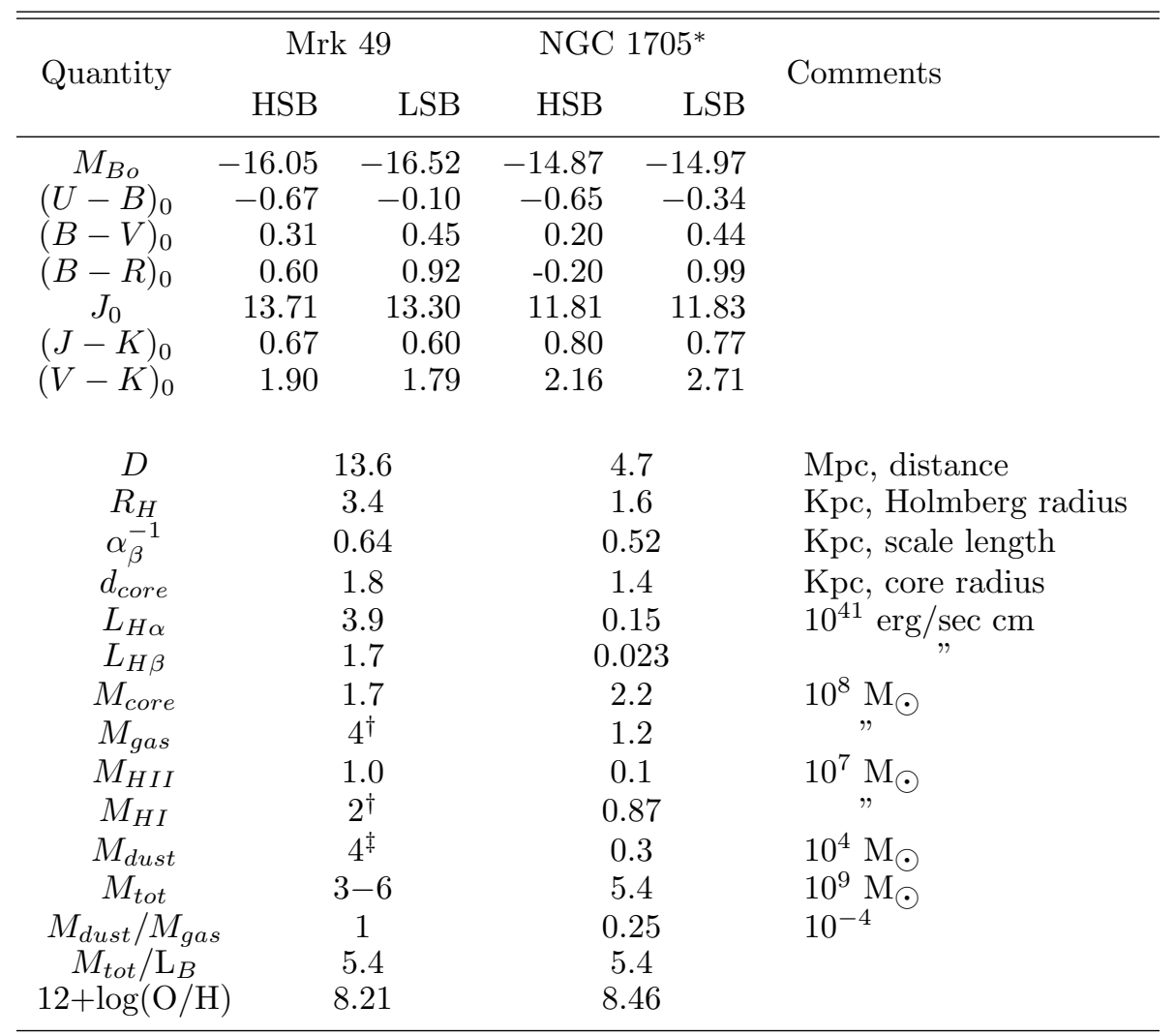

* adopted from Meurer et al.(1992)

$\dagger$ adopted from Thuan \& Martin (1981)

$\ddagger$ adopted from Parravano(1989)

Bothun, G. D., Mould, J. R., Caldwell, N., \& MacGillivary, H. T., 1986, Comparative photometric parameters of dwarf irregular and elliptical galaxies in the Virgo Cluster - Two different classes of dwarf galaxies?, AJ, 92, 1007

Bucherhorn, 1992, PhD thesis, Australian National University

Cairós, L.M., Víchez, J.M., González, P., José, N., Iglesias-Parámo, J. \& Caon, N. 2001, Multiband Analysis of a Sample of Blue Compact Dwarf Galaxies. I. Surface Brightness Distribution, Morphology, and Structural Parameters, ApJS, 133, 321

Campbell, A.W. \& Terlevich, R., 1984, The origin of the infrared luminosity in violent star formation regions, MNRAS, 211, 15

Deharveng, J.-H., Albrecht, R., Babieri, C., Blades, J. C., Boksenberg, A., Crane, P., Disney, M. J., Jakobsen, P., Kamperman, T. M., King, I. R., Macchetto, F., Mackay, C. D., Paresce, F., Weigelt, G., Baxter, D., Greenfield, P., Jedrzejewski, R., Nota, A., \& Sparks, W. B., 1994, The massive star content of the blue dwarf galaxy IZw 36 from Faint Object Camera observations, A\&A, 288, 413

Dinerstein, H. L. \& Shields, G. A., 1986, Reassessing the primordial helium abundance - New observations of NGC 4861 and CG 1116 + 51, ApJ, 311, 45

Doherty, R. M., Puxely, P. J., Lumsden, S. L., \& Doyon, R., 1995, Helium and hydrogen excitation in starburst galaxies - infrared and optical line ratios, MNRAS, 277, 577

Drinkwater, M. \& Hardy, E., 1991, Extreme blue compact dwarf galaxies in the Virgo Cluster, AJ, 101, 94

Driver, S. P., Phillipps, S., Davies, S. P., Morgan, I., \& Disney, M. J., 1994, Dwarf Galaxies at Z=0.2 - Photometry of the Cluster ABELL:963, MNRAS, 268, 393

Ellis, R.S., 1997, Faint Blue Galaxies, ARA\&A, 35, 389

English, J., 1994, PhD thesis, Australian National University

Freeman, K. C., 1992, Dark matter in galaxies in Thuan, T. X. et al., eds, Physics of Nearby Galax- 
ies: Nature or Nurture? Editions Frontieres, Gifsur-Yvette, p.201

Gallagher III, J. S. \& Hunter, D. E., 1986, UBV colors of Virgo cluster irregular galaxies, AJ, 92, 557

Gil de Paz, A., Madore, B. F., \& Pevunova O., 2003, Palomar/Las Campanas Imaging Atlas of Blue Compact Dwarf Galaxies. I. Images and Integrated Photometry, ApJS, 147, 29

Graham, J. A., 1982, UBVRI Standard Stars in the E-Regions, PASP, 94, 244

Gordon, D. \& Gottemann, S. T., 1981, H I observations of blue compact galaxies, AJ, 86, 161

Guzman, R., Jargren, A., Koo, D. S., Bershady, M. A., \& Simard, L., 1998, Optical Structure and Colors of Faint Compact Narrow Emission-Line Galaxies, ApJ, 495, 13

Hodapp, K-W, Rayner, J., \& Irwin, E., 1992, The University of Hawaii NICMOS-3 near-infrared camera, PASP, 104, 441

Hunter, D. A., Gallagher III, J. S., Rice, W. L., \& Gillett, F. C., 1989, IRAS observations of a large sample of normal irregular galaxies, ApJ, 336, 152

Hunter, D. A. \& Gallagher III, J. S., 1985, Star-forming properties and histories of dwarf irregular galaxies Down but not out, ApJS, 58, 533

Hunter, D. A. \& Thronson Jr, H. A., 1995, The Massive Stars of I ZW 18 as Seen in Hubble Space Telescope Images, ApJ, 452, 238

Huchra, J., 1977, The nature of Markarian galaxies, ApJS, 35, 171

James, P., 1991, An infrared study of dwarf galaxies in the Virgo cluster, MNRAS, 250, 544

Joy, M. \& Lester, D. F., 1988, Near-infrared line and continuum emission from the blue dwarf galaxy II ZW 40, ApJ, 331, 145

Joyce, R. R., 1992, ASP Conference Series, Vol 23, (ed. by Steve B. Howell), 258

Knapp, G. R., Turner, E. L., \& Cunniffe, P. E., 1985, The statistical distribution of the neutral-hydrogen content of elliptical galaxies, AJ, 90, 454

Kobulnicky, H. A., Dickey, J. M., Sargent, A. I., Hogg, D. E. \& Conti, P. S., 1995., Aperture Synthesis Observations of Molecular and Atomic Gas in the WolfRayet Starburst Galaxy, AJ, 110, 116

Lake, G. \& Schommer, R. A., 1984, A successful survey of H I in low-luminosity elliptical galaxies, ApJ, 280, 107

Lake, G., Schommer, R. A., \& van Gorkom, J. H., 1987, The H I distribution and kinematics in four lowluminosity elliptical galaxies, ApJ, 314, 57

Landolts, A. U., 1992, Optical imaging of QSOs with 0.5 arcsec resolution, AJ, 104, 1
Mackie, G. H., 1989, PhD Thesis, Australian National University

McCall, M. L., 1984, Emission coefficients for gaseous nebulae - Three-level atom approximations, MNRAS, 208, 253

Melnick, J., Heydari-Malayeri, M. \& Leisy, P., 1992, The metal-poor H II galaxy SBS 0335 - 052 and the primordial helium abundance, A\&A, 253, 16

Mendoza, C., 1983, Planetary Nebulae ed. by D. R. Flower(Dordrecht: Reidel) p.143

Meurer, G. R., Freeman, K. C., Dopita, M. A. \& Cacciari, C., 1992, NGC 1705. I - Stellar populations and mass loss via a galactic wind, AJ, 103, 60

Meurer, G. R., Mackie, G., \& Carignan, C., 1994, Optical observations of NGC 2915: A nearby blue compact dwarf galaxy, AJ, 107, 2021

Mihos, J. C. \& Hernquist, L., 1994, Dense stellar cores in merger remnants, ApJ, 437, 47

Noeske, K. G., Iglesias-Páramo, J., Vilchez, J. M., Papaderos, P., \& Fricke, K. J., 2001, On faint companions in the close environment of star-forming dwarf galaxies. Possible external star formation triggers?, A\&A, 371, 806

Noeske, K. G., Papaderos, P., Cairós, L. M., \& Fricke, K. J., 2005, New insights to the photometric structure of Blue Compact Dwarf Galaxies from deep near-infrared studies. II. The sample of northern BCDs, A\&A, 429, 115

O'Connell, R. W., Gallagher III, J. S., \& Hunter, D. A., 1994, Hubble Space Telescope imaging of superstar clusters in NGC 1569 and NGC 1705, ApJ, 433, 65

Östlin, G., Amram, P., Bergvall, N., Masegosa, J., Boulesteix, J., \& Marquez, I., 2001, Dynamics of blue compact galaxies, as revealed by their $\mathrm{H}$ velocity fields. II. Mass models and the starburst triggering mechan, A\&A, 374, 800

Osterbrock, D. E., 1975, Astrophysics of Gasouse Nebulae, W. H. Freeman and Company: Sanfransisco,

Papaderos, P., Guseva, N. G., Izotov, Y. I. Noeske, K. G., Thuan, T. X., \& Frcke, K. J., 2006, New southern blue compact dwarf galaxies in the $2 \mathrm{dF}$ Galaxy redshift survey, A\&A, 457, 45

Parravano, A., 1989, A self-regulated star formation rate as a function of global galactic parameters, ApJ, 347,812

Persson, S. E., Aaronson, M., Cohen, J. G., Frogel, J. A., \& Mathews, K., 1983, Photometric studies of composite stellar systems. V - Infrared photometry of star clusters in the Magellanic clouds, ApJ, 266, 105

Pisano, D. J., Kobulnicky, H. A., Guzman, R., Galleo, J., \& Bershady. M. A., 2001, The Gas Content and Kinematics of Nearby Blue Compact Galaxies: Implications for Studies at Intermediate and High Redshift, AJ, 122, 1194 
Pustilnik, S. A., Knuazev, A. Y., Lipovesky, V. A., \& Ugryumov, A. V., 2001, Environment status of blue compact galaxies and trigger of star formation, A\&A, 373, 24

Quillen, A. C., Ramirez, S. V., \& Frogel, J. A., 1995, The Dwarf Galaxy NGC 1705-A Highly Composite Stellar Population, AJ, 110, 205

Ryder, S. D., 1993, PhD thesis, Australian National University

Schweizer, F. \& Seitzer, P., 1992, Correlations Between UBV Colors and Fine Structure in E+SO Galaxies, IAUS, 149, 488

Seaton, M. J., 1979, Interstellar extinction in the UV, MNRAS, 187, 73

Shi, F., Kong, X., \& Cheng, F. Z., 2005, Spectroscopic study of blue compact galaxies. V. Oxygen abundance and the metallicity-luminosity relation, A\&A, 437,849

Shostak, G. S. \& van der Kruit, P. C., 1984, Studies of nearly face-on spiral galaxies. II - H I synthesis observations and optical surface photometry of NGC 628, A\&A, 132, 20

Stavely-Smith, L., Davies, R. D., \& Kinman, T. D., 1992, H I and optical observations of dwarf galaxies, MNRAS, 258, 334

Struck-Marcell, C. \& Tinsley, B. H., 1978, H I and optical observations of dwarf galaxies, ApJ, 221, 562

Stone, R. P. S. \& Baldwin, J. A., 1983, Southern spectrophotometric standards for large telescopes, MNRAS, 204, 347

Sung, E.-C., Chun, M.-S., Park, J.-H., \& Moon, H.-K., 1996, Southern Blue Compact Dwarf Galaxy ESO 105-IG11 II.: Abundance Analysis, JASS, 13, 91

Sung, E.-C., Han, C., Ryden, B., Patterson, R. J., Chun, M.-S., Kim, H.-I., Lee, W.-B., \& Kim, D.-J., 1998, The Intrinsic Shapes of Low Surface Brightness Dwarf Irregular Galaxies and Comparison to Other Types of Dwarf Galaxies, ApJ, 505, 199

Sung, E.-C., 2000, Spectroscopic and Photometric Studies of Blue Compact Dwarf Galaxies, PASP, 112, 121 (Dissertation Summary)

Sung, E.-C., Chun, M.-S., Freeman, K. C., \& Chaboyer, B., 2002, A New Classification Scheme for Blue Compact Dwarf Galaxies, ASPC, 273, 341

Thuan, T. X., 1983, Blue compact dwarf galaxies. II Near-infrared studies and stellar populations, ApJ, 268,667

Thuan, T. X, Izotov, Y. I., \& Lipovesky, V. A., 1996, Hubble Space Telescope Observations of the Unusual Blue Compact Dwarf Galaxy Markarian 996, ApJ, 463, 120

Thuan, T. X. \& Martin, G. E., 1981, Blue compact dwarf galaxies. I - Neutral hydrogen observations of 115 galaxies, ApJ, 247, 823
Vanzi, L., Rieke, G. H., Martin, C. L., \& Shield, J. C., 1996, The History of Star Formation in II ZW 40, ApJ, 466, 150

van Zee, L., Skillman, E. D., \& Salzer, J. J., 1998, Neutral Gas Distributions and Kinematics of Five Blue Compact Dwarf Galaxies, AJ, 116, 1186

Vilchez, J. M. \& Igleisias-Páramo, J., 2003, Spectroscopy of Dwarf Galaxies in the Virgo Cluster. I. Data, Chemical Abundances, and Ionization Structure, ApJS, 145, 225

Wainscoat, R. J. \& Cowie, L. L., 1992, A filter for deep near-infrared imaging, AJ, 103, 332 